\title{
Resonance properties of domain boundaries in quasi-two dimensional antiferromagnets
}

\author{
A. S. Kovalev, J. E. Prilepsky, E. A. Kryukov, and N. V. Kulik
}

Citation: Low Temperature Physics 36, 831 (2010); doi: 10.1063/1.3493440

View online: https://doi.org/10.1063/1.3493440

View Table of Contents: http://aip.scitation.org/toc/ltp/36/8

Published by the American Institute of Physics

\section{Articles you may be interested in}

Antiferromagnetic resonance in $\mathrm{Mn}_{2} P_{2} S_{6}$

Low Temperature Physics 35, 930 (2009); 10.1063/1.3272560

LOW TEMPERATURE TECHNIQUES OPT|CAL CAVITV PUIYSI|CS

MITIGATING THERMAL \& VIBRATIONAL NOISE

\section{DOWNLOAD THE WHITE PAPER}




\title{
Resonance properties of domain boundaries in quasi-two dimensional antiferromagnets
}

\author{
A. S. Kovalev ${ }^{\text {a) }}$ \\ B. I. Verkin Institute of Low Temperatures (FTINT), National Academy of Sciences of Ukraine, pr. Lenina \\ 47, Kharkov 61103, Ukraine and V. N. Karazin Kharkov National University, pl. Svobody 4, Kharkov \\ 61077, Ukraine \\ J. E. Prilepsky \\ B. I. Verkin Institute of Low Temperatures (FTINT), National Academy of Sciences of Ukraine, pr. Lenina \\ 47, Kharkov 61103, Ukraine and Nonlinearity and Complexity Research Group, School of \\ Engineering and Applied Sciences, Aston University, Aston Triangle, Birmingham, United Kingdom
}

\section{E. A. Kryukov and N. V. Kulik}

V. N. Karazin Kharkov National University, pl. Svobody 4, Kharkov 61077, Ukraine (Submitted April 6, 2010)

Fiz. Nizk. Temp. 36, 1041-1049 (August-September 2010).

The so-called "internal modes" localized near the domain boundaries in quasi-two dimensional antiferromagnets are investigated. The possible localized states are classified and their frequency dependences on the system discreteness parameter $\lambda=J / \beta$, which describes the ratio of the magnitudes of the exchange interplane interaction and the magnetic anisotropy, are found. A sudden change in the spectrum of the local internal modes is observed at a critical value of this parameter, $\lambda=\lambda_{b}=3 / 4$, where the domain wall shifts from a collinear to a canted shape. When $\lambda<\lambda_{b}$ there are one symmetric and two antisymmetric local modes, and when $\lambda>\lambda_{b}$ the modes are two symmetric, one antisymmetric, and one shear. For discreteness parameters close to the critical value, the frequencies of some of the local modes lie deep inside the gap for the linear AFM magnon spectrum and can be observed experimentally. (c) 2010 American Institute of Physics. [doi:10.1063/1.3493440]

Current advances in the physics of magnetic phenomena are related substantially to experimental studies of new magnetic materials with complicated internal structures. Many of these substances are low-dimensional: quasi-two dimensional and quasi-one dimensional. ${ }^{1,2}$ Examples include HTSC compounds, two-dimensional magnets based on Langmuir-Blodgett films, and metal-organic lowdimensional magnets. The latter have been intently studied experimentally for more than 20 years in a number of laboratories, including the Institute of Low Temperatures of the National Academy of Sciences of Ukraine (FTINT NANU), where a comprehensive study of their resonance properties has been under way. Layered magnets ${ }^{3-5}$ are of special interest to experimentalists and theorists. The materials have a great variety of physical properties: the interactions within layers and between layers, as well as the interlayer exchange interaction, can be ferromagnetic (FM) and antiferromagnetic (AFM), and the energy of the magnetic anisotropy has a different character. By varying the structure of these magnets it has been possible to attain conditions such that the exchange interaction between the magnetic layers became much smaller than the exchange interaction within the planes $\left(J \ll J_{\|}\right)$and approached the order of magnitude of the singleion (or exchange) anisotropy $J \sim \beta$ or even much smaller than the later. ( $\mathrm{J}$ is the exchange interlayer interaction constant and $\beta$ is the single-ion anisotropy constant.) The parameter $\lambda=\left(l_{0} / a\right)^{2}$, where $l_{0}=a \sqrt{J / \beta}$ is the scale length for the spatial inhomogeneity in the magnetization (e.g., the width of a domain wall- the "magnetic length") and a is the lattice constant, characterizes the discreteness of the system. The limit $\lambda<1$ is customarily referred to as the "anticontinuum limit" and research on the nonlinear dynamics of physical systems in this limit is often among the most interesting areas in modern theoretical physics. ${ }^{6}$ In particular, many metal-organic antiferromagnets ${ }^{1-5}$ with $\left(\left(\mathrm{C}_{n} \mathrm{H}_{2 n+1}\right)\right.$ $\left.\times\left(\mathrm{NH}_{3}\right)\right)_{2}\left(\mathrm{XCl}_{4}\right)$ and $\left(\left(\mathrm{NH}_{3}\right)\left(\mathrm{CH}_{2}\right)_{n}\left(\mathrm{NH}_{3}\right)\right)\left(\mathrm{XCl}_{4}\right)$ structures with magnetic copper and manganese $(\mathrm{X})$ atoms in layers with a different number $\mathrm{n}$ of organic $\mathrm{CH}_{2}$ groups in the molecules intercalating the layers of magnetic atoms, as well as some other substances with magnetic iron ions ${ }^{7}$ and inorganic intercalants, ${ }^{8}$ have these kinds of discreteness properties in the direction perpendicular to the plane of the magnetic layers. These substances have clearly distinct nonlinear properties. Examples include the nonlinear AFM resonance, ${ }^{9}$ proof of the existence of "magnetic skyrmions," namic magnetic solitons ("breathers") ${ }^{12}$ discovered in them. The experimental observation of the so-called "exchange mode" should also be mentioned. ${ }^{13}$ It is important that, in the case of these layered metal-organic antiferromagnets, the number $\mathrm{n}$ can vary, so that the dependence of the structure and dynamic properties of these magnets on the magnitude of the interlayer exchange integral $J$, i.e., on the discreteness parameter of the system, can be studied experimentally. This has made it possible to trace the transition from threedimensional to quasi-two dimensional behavior in their physical properties. (Of course, it is necessary to keep in mind that the properties change greatly when $n$ is changed, even by unity.) 
During some resonance experiments with quasi-two dimensional AFMs an additional (besides the homogeneous AFM resonance and exchange mode bands) RF absorption band was discovered lying below the homogeneous AFM resonance (below the limit of the linear wave spectrum of the AFM magnons) in the depth of the gap for the spin wave spectrum. ${ }^{7,8,14-16}$ Since the experiments were done at low pump levels and the additional absorption peaks were located in the depth of the gap for the magnon spectrum, the observation of these absorption peaks could be explained by the existence of linear modes localized near existing nonlinear magnetic defects, primarily next to domain walls. This was confirmed directly in a paper ${ }^{8}$ where the phenomenon was observed only after rapid cooling of the sample to liquid helium temperature (over $20 \mathrm{~min}$ ) and not observed after slow cooling (over $3 \mathrm{~h}$ ). The different cooling rate leads to the appearance of a different density of structural defects such as domain walls or magnetic layers with magnetization in an reversed direction compared to an ideal AFM structure. This kind of defect can be represented as a bound state of two domain walls, so it is natural to refer to it as a soliton defect. (Because of the considerable difference in the exchange interaction within and between layers, we speak of walls and layers with "inverted spins," parallel to the magnetic layers.

In metal-organic compounds an absorption peak in the forbidden region of the spectrum has only been observed for a few values of $n$ such that the discreteness parameter $\lambda$ is on the order of unity and is not observed at small and large values of $\mathrm{n}$. This indicates that the frequency dependence of the internal modes on the parameter $J / \beta$ (interlayer exchange interaction) should undergo a sharp change when $J$ $\sim \beta$.

It has been correctly suggested ${ }^{14-16}$ that additional absorption peaks may be associated with "internal modes" of essentially discrete domain walls and magnetic layers with inverted spins, as confirmed by analytic calculations. ${ }^{17}$ These calculations ${ }^{17}$ were done for the case of a ferromagnetic interaction between layers and anomalously small values of the discreteness parameter $(\lambda<3 / 4)$ at which the domain wall acquires a collinear shape. Later came theoretical studies ${ }^{18-20}$ of the dependence of the frequency of the localized mode on the magnitude of the interlayer interaction with arbitrary values of the discreteness parameter. However, as in Ref. 17, there the interaction between the magnetic layers was assumed to be ferromagnetic, while this interaction was typically antiferromagnetic in the experiments. Since experimental studies of new series of samples of quasi-two dimensional magnets are continuing and new data are being obtained on the existence of localized modes with frequencies within the gap of the spectrum, theoretical studies of this problem continue to be of interest for the real case of an antiferromagnetic exchange interaction between the magnetic layers. This paper is also devoted to this problem; here we study the structure of the eigenmode spectrum of a homogeneous antiferromagnetic chain in the presence of a domain wall.

There is yet another motive for our paper. For more than 20 years, internal modes of topological excitations in nonlinear dynamic systems of different kinds have been the object of abiding interest for theorists involved in the study of soli- ton dynamics. A large number of papers have examined the properties of internal modes in continuous systems (close to integrable) with different forms of linear wave dispersion (V. G. Bar'yakhtar and colleagues have made a major contribution to solving this problem). ${ }^{21-26}$ Internal modes have also been studied in a substantially discrete limit in FrenkelKontorova models with a linear interparticle interaction (e.g., the DSGE-discrete sinusoidal Gordon equation). ${ }^{27-29}$ In these cases the frequencies of the internal modes of the domain walls are usually weakly detached from the boundary of the linear wave spectrum, even in the anticontinuum case. In magnetically ordered media the interaction between magnetic moments is essentially nonlinear, and this leads to the formation of collinear structures and to a radical change in the frequency characteristics of the internal modes. ${ }^{30} \mathrm{We}$ note that, as applied to magnetic systems, internal modes and more complicated objects than topological defects have been examined, in particular the spectra of antiferromagnetic semiconductors (taking the coupling to the electronic subsystem into account) when magnetic polarons with different structures are present. ${ }^{31}$

\section{MODEL FORMULATION AND BASIC EQUATIONS}

Since the exchange magnetic interaction within the magnetic layers is considerably greater than the interlayer interaction (for example, in the $\left(\mathrm{C}_{n} \mathrm{H}_{2 n+1} \mathrm{NH}_{3}\right)_{2} \mathrm{CuCl} 4$ series of compounds, ${ }^{20}$ the ratio of the exchange interactions is $\left|J / J_{\|}\right|=10^{-3}$ for $n=1$ and $\left|J / J_{\|}\right|=10^{-20}$ for $n=10$ ), in a study of the interlayer interaction the entire magnetic layer can be regarded as a single large magnetic moment viewed classically. The magnetic dynamics can be treated in terms of the Landau-Lifshitz equations ${ }^{28}$ for a one-dimensional Heisenberg model with an energy of the form

$$
E=\sum_{n}\left[J \mathbf{S}_{n} \mathbf{S}_{n+1}-\frac{\beta}{2}\left(\mathbf{S}_{n} \mathbf{e}_{z}\right)^{2}\right],
$$

where $\mathbf{S}_{n}$ is the point magnetic moment ("spin") and for AFMs $J>0$ is the exchange between layers. We limit ourselves to the simplest case of a uniaxial magnetic anisotropy with $\beta>0$ for an easy-axis AFM with the easy axis directed along the $\mathbf{e}_{z}$ axis. Neglecting damping, here the LandauLifshitz equation can be written in the form

$$
\frac{1}{\omega_{0}} \frac{d \mathbf{S}_{n}}{d t}-\lambda\left[\mathbf{S}_{n},\left(\mathbf{S}_{n+1}+\mathbf{S}_{n-1}\right)\right]+\left[\mathbf{S}_{n}, \mathbf{e}_{z}\right]\left(\mathbf{S}_{n}, \mathbf{e}_{z}\right)=0
$$

where $\omega_{0}=2 \beta \mu_{0} S_{0} / \hbar$ is the frequency of the homogeneous AFM resonance, $\mu_{0}$ is the Bohr magneton, $S_{0}$ is the nominal magnetization of a unit cell, and $\lambda=\left(l_{0} / a_{0}\right)^{2}$ is the discreteness parameter associated with the magnetic length mentioned above. (In the following, time is measured in units of $1 / \omega_{0}$.) The parameter $n$ enumerates the magnetic layers in the $X$ direction. (In the exchange approximation, this direction is not connected in any way with the easy axis $Z$.) When $\lambda \sim 1$ the lattice becomes substantially discrete in the $X$ direction, while $\lambda \gg 1$ corresponds to the long-wave limit. Note that the structure of the ground state and the states that are inhomogeneous along the $X$ axis, as well as the classification of the local modes in the limit of large intralayer exchange, 

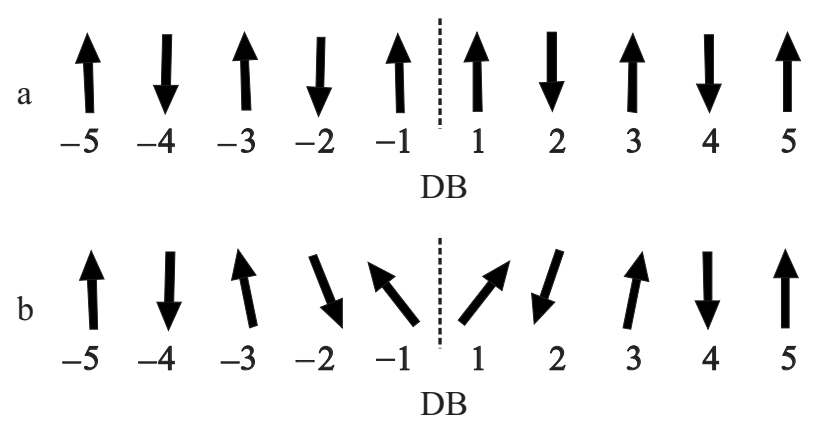

FIG. 1. The structures of collinear (a) and canted (b) AFM domain walls.

are independent of the type (AF or AFM) of magnetic ordering in the magnetic layers.

The structure of the static states of the system (both the ground state and the inhomogeneous states) is the same for FM and AFM interactions between layers (it is necessary only to change the direction of the even moments to the opposite) and has been studied in Refs. 17-20. (The dynamics in these cases is, of course, different.)

In the ground state the moments of the layers are oriented along the easy axis anticollinearly and it is convenient to shift to the complex field variable $\psi_{n}=S_{n}^{x}+i S_{n}^{y}$ (the classical analog of the creation operator for magnons) and the projection on the $Z$ axis, $Z: S_{n}^{z} \equiv m_{n}$. Here the condition $\left|\psi_{n}\right|^{2}+m_{n}^{2}=1$ is satisfied (we assume the nominal magnetization to be equal to unity, i.e., $\left.S_{0}=1\right)$ and Eq. (1.2) can be rewritten in the form

$i \frac{d \psi_{n}}{d t}-\lambda\left(\psi_{n} m_{n+1}-\psi_{n+1} m_{n}+\psi_{n} m_{n-1}-\psi_{n-1} m_{n}\right)+\psi_{n} m_{n}=0$,

where the discreteness of the system is characterized by the parameter $\lambda=(J / \beta)$.

\section{STATIC STRUCTURES OF COLLINEAR AND CANTED DOMAIN WALLS}

We begin by studying the static configurations of the domain walls and note that Eq. (1.3) allows a time independent solution for a collinear domain boundary (Fig. 1a) with the following orientation of the spins:

$$
m_{2 n}=-1 ; \quad m_{2 n+1}=1, \quad \psi_{n}=0,
$$

where $\mathrm{n}$ is an integer. (For convenience the spins are enumerated as shown in Fig. 1a, where there is no $n=0$ and the domain boundary (DB) lies between the spins with numbers -1 and 1 .

For values of the exchange interaction large than critical $\left(\lambda>\lambda_{b}=3 / 4\right)$, besides domain boundaries with a collinear structure that correspond to solutions of Eq. (2.1), canting of the boundary becomes possible with a noncollinear distribution of spins within the domain wall: $m_{n} \neq \pm 1$. (See Fig. $1 b)$. This transition takes place as a bifurcation.

To find the bifurcation point we linearize Eq. (1.3) in the time independent case with respect to the small deviations $\psi_{n}$ of the magnetization vectors from a collinear configuration. Symmetry considerations imply that $\psi_{n}=-\psi_{-n}$ and $m_{n}=m_{-n}$. For moments with $n>0$ we obtain the system of linear difference equations

$$
\begin{aligned}
& (1-\lambda) \psi_{1}+\lambda \psi_{2}=0, \\
& (1+2 \lambda) \psi_{n}+\lambda\left(\psi_{n+1}+\psi_{n-1}\right)=0, \quad n>1,
\end{aligned}
$$

whose solution for $n \geqslant 1$ has the simple form

$$
\psi_{n}=(-1)^{n} a / 3^{1-n}, \quad \lambda=\lambda_{b}=3 / 4,
$$

where the constant a is arbitrary in the first iteration of perturbation theory and is found in the second approximation. It is easy to obtain an approximate solution near the bifurcation point using the asymptotic procedure ${ }^{32}$ of expanding with respect to the small parameter

$$
\varepsilon=\lambda-\lambda_{b},
$$

which characterizes the deviation of the discreteness parameter $\lambda$ from its value at the bifurcation. We write the approximate solution (1.3) in the form

$$
\psi_{n}=(-1)^{n}\left(a^{(1)} \mathrm{e}^{-\nu(n-1)}+a^{(3)} \mathrm{e}^{-3 \nu(n-1)}+\ldots\right),
$$

where $a^{(1)} \sim \varepsilon^{1 / 2}, a^{(3)} \sim \varepsilon^{3 / 2}$, etc. After substituting Eq. (2.6) in Eqs. (1.3), including the boundary conditions, we find that $\cosh \nu=1+1 / 2 \lambda$ (i.e., in the first approximation with $\lambda$ $=3 / 4$ we have $\exp \nu=3$, which corresponds to the bifurcation point found above) and $a^{(3)}=a^{(1) 3} \lambda(1+2 \lambda) / 2(1+4 \lambda)$ $\approx 15 a^{(1) 3} / 64$. In the first approximation the amplitude $a$ $\equiv a^{(1)}$ of the solution depends on the discreteness parameter as follows:

$$
a^{2}=72 \varepsilon / 37 \approx 2 \varepsilon .
$$

Therefore, the static configuration of the canted domain boundary is given by

$$
\psi_{n}^{(0)}=-\psi_{-n}^{(0)} \approx(-1)^{n} \sqrt{2} \frac{\sqrt{\lambda-\lambda_{b}}}{3^{n-1}}, \quad n \geqslant 1 .
$$

Note that the amplitude of the solution near the bifurcation point becomes smaller as the discreteness parameter deviates from the bifurcation value and falls off rapidly with distance form the center of the domain wall. As the parameter $\lambda$ rises, the width of the domain wall increases.

Substituting the solutions (2.1) and (2.8) in Eq. (1.1) for the system energy, we find $E=E(\lambda)$ for collinear and canted walls (relative to the ground state energy) to be

$$
\begin{aligned}
& E_{\mathrm{col}} / \beta=2 \lambda, \\
& \frac{E_{\mathrm{can}}}{\beta} \approx 2 \lambda-\lambda_{b}\left(\lambda-\lambda_{b}\right), \quad 0<\frac{\lambda-\lambda_{b}}{\lambda_{b}} \ll 1 .
\end{aligned}
$$

As will be shown below, a collinear domain wall structure remains stable only to a critical value of the discreteness parameter $\lambda=\lambda_{\mathrm{c}}=3 / 2$, while a canted structure undergoes a change with an increase in this number. Near the bifurcation point the energy of a canted wall is equal to $E_{\text {can }} / \beta \approx 9 / 16$ $+5 \lambda / 4$.

We now examine the domain wall structure in the long wave limit corresponding to discreteness parameters $\lambda \gg 1$. In the time independent case, Eq. (1.3) reduces to the standard form 


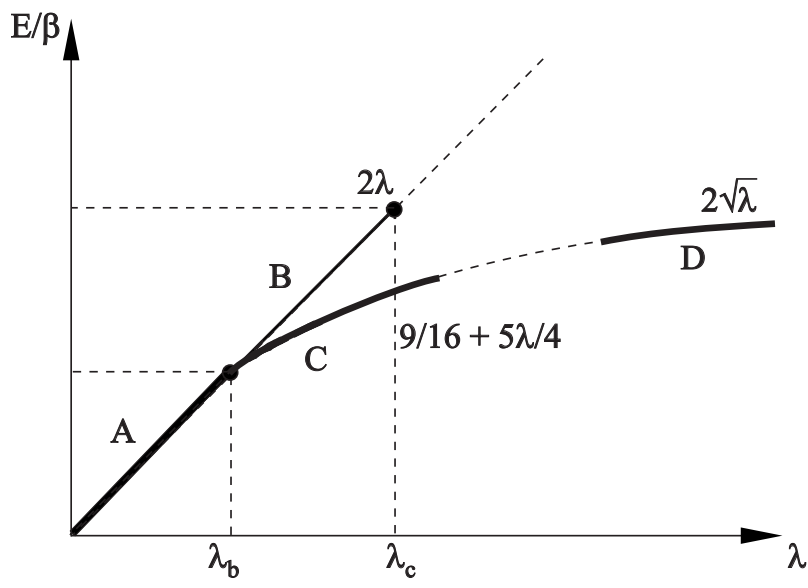

FIG. 2. The normalized domain wall energy for different configurations as a function of the discreteness parameter. (Shown here are plots of $E(\lambda)$ for a collinear wall structure (A, B), a canted wall near the bifurcation point $(C)$, and the asymptote in the long-wave limit (D).

$$
2 \lambda d^{2} \theta / d x^{2}=\sin 2 \theta
$$

for the angle $\theta$ by which the antiferromagnetism vector deviates from the easy axis. Its solution for a domain boundary, $\theta=2 \arctan \exp (x / \sqrt{\lambda})$, corresponds to the asymptote of the energy as a function of the discreteness parameter, $E / \beta$ $=2 \sqrt{\lambda}$. All the dependences of the domain wall energy on $\lambda$ found above are shown in Fig. 2. It can be seen that the asymptotes of the domain boundary energies in the different configurations as functions of the discreteness parameter all "match" well.

\section{THE SPECTRUM OF THE INTERNAL MODES OF COLLINEAR DOMAIN WALLS IN THE ANTICONTINUUM LIMIT}

The spectrum of linear spin waves in a two-sublattice AFM in a uniform ground state has the well known form ${ }^{28}$

$$
\omega= \pm \sqrt{(1+4 \lambda)+4 \lambda^{2} \sin ^{2} k}
$$

where $\mathrm{k}$ is the wave vector in the direction perpendicular to the plane of the layers. As opposed to the ferromagnetic case, this spectrum is symmetric with respect to changes in the sign of the frequency and differs substantially from the expression for FM ordered layers, $\omega_{f}=1+4 \lambda \sin ^{2}(k / 2)$. Thus, in our notation the gap in the spectrum of linear AFM magnons changes in the following way as the discreteness parameter (interlayer exchange) is varied:

$$
\omega_{\min }=\sqrt{1+4 \lambda} \text {. }
$$

(The upper boundary of the spectrum, the so-called "exchange mode" is given by $\omega_{\max }=1+2 \lambda$.) In Fig. 3 the region of the magnon spectrum is shaded.

We begin our discussion of the internal modes of domain walls with the case of collinear boundaries with the magnetic moment distribution given by Eq. (2.1). On substituting it in the dynamical equations (1.3), for the stationary solutions of the form $\psi_{n}=a_{n} \exp (i \omega t)$ with $\mathrm{n}>0$ we obtain the system of equations

$$
(1+2 \lambda+\omega) a_{2 n}+\lambda\left(a_{2 n+1}+a_{2 n-1}\right)=0,
$$

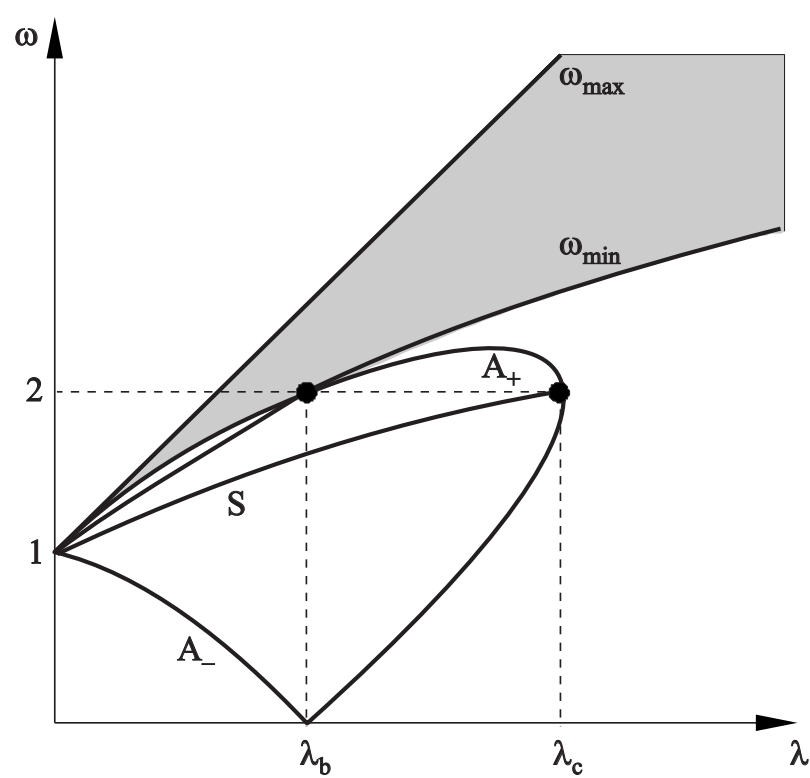

FIG. 3. The dependence of the spectrum of the linear magnons and internal modes of a collinear domain wall on the discreteness parameter (all the eigenfrequencies are shifted into the positive frequency region.

$$
(1+2 \lambda-\omega) a_{2 n+1}+\lambda\left(a_{2 n+2}+a_{2 n}\right)=0
$$

with following the boundary condition at the domain boundary:

$$
(1 \pm \lambda-\omega) a_{1}+\lambda a_{2}=0
$$

for symmetric $(+)$ internal modes with $a_{n}=a_{-n}$ and antisymmetric modes (-) with $a_{n}=-a_{-n}$, respectively. (As opposed to a ferromagnetic collinear $\mathrm{DB},{ }^{17}$ in the case of an antiferromagnet several internal localized modes can exist with different symmetries.) The internal modes correspond to walllocalized solutions of the form

$$
a_{2 m}=A \exp (-2 m \xi), \quad a_{2 m+1}=B \exp (-(2 m+1) \xi)
$$

with different rotation amplitudes for differently directed moments. Here the dependence of the frequency of the internal modes on the localization parameter is given by

$$
\omega= \pm \sqrt{(1+4 \lambda)-4 \lambda^{2} \sinh ^{2} \xi}
$$

which obviously follows from Eq. (3.1) on replacing the magnon wave number by an imaginary quantity $k \rightarrow i \xi$, which determines the localization region for the internal mode. The ratio of the rotation amplitudes of differently directed moments is given by $A=-B 2 \lambda \cosh \xi /(1+2 \lambda+\omega)$. Given this equation and substituting Eqs. (3.5) and (3.6) in the boundary conditions (3.4), we obtain the following dependence of the frequency of the symmetric internal mode on the discreteness parameter:

$$
\omega= \pm \sqrt{1+2 \lambda}
$$

which is plotted as curve $\mathrm{S}$ in Fig. 3. The localization region for the symmetric mode $l \sim 1 / \xi$ decreases for small $\lambda$ as $l$ $\sim-1 / \ln \lambda$ and increases for large $\lambda$ as $l \sim \lambda^{1 / 2}$. The ratio of the rotation amplitudes of the moments is $A / B=(1$ $-\sqrt{1+2 \lambda}) / \sqrt{2 \lambda}$.

Besides the symmetric modes, there are also two antisymmetric modes with $a_{n}=-a_{-n}$. In this case, the boundary 
condition (which differs from that given above for the symmetric mode) yields these dependences for the frequencies $\omega$ of the localized modes on the discreteness parameter $\lambda$ :

$$
\omega_{ \pm}=\left(-4 \lambda \pm \sqrt{9+6 \lambda-8 \lambda^{2}}\right) / 3 .
$$

Equations (3.7) and (3.8) show that the two branches of the $\omega(\lambda)$ curve for the symmetric internal modes are symmetric with respect to the sign of the frequency, while for the antisymmetric internal modes the two branches (3.8) have different dependences on the discreteness parameter owing to the finite magnetization of the domain wall, which leads to an asymmetry in directions relative to the easy axis. On the other hand, when the center of an AFM domain wall is shifted by one interatomic distance, the nonzero magnetization of the domain boundary changes its sign to the opposite. Here the frequencies of all the eigenstates of the system change sign. Thus, it is possible to change the sign of the frequency in the negative frequency solutions and reflect the entire spectrum of the oscillations into the region $\omega \geqslant 0$. These dependences are shown in Fig. 3 as the curves $A_{+}$and $A_{-}$. Equation (3.8) for $\omega_{+}$with small values of the discreteness parameter splits slightly away from the lower boundary of the continuous spectrum, and at $\lambda=\lambda_{\mathrm{b}}$ becomes tangent to it. (At the bifurcation point $\lambda=\lambda_{\mathrm{b}}$ the frequency of the upper antisymmetric mode is $\omega=2$, while the frequency of the symmetric mode is $\omega=\sqrt{5 / 2}$.) Equation (3.7) for the symmetric internal mode detaches more strongly from the boundary. Finally, the strongest detachment from the frequency of the AFMR is observed for the antisymmetric mode $\omega_{-}$(38). The dependence of the frequency on $\lambda$ for this mode goes to zero at $\lambda=\lambda_{\mathrm{b}}$. For large $\lambda>\lambda_{\mathrm{b}}$ the absolute minimum of the energy corresponds to a canted DB shape, but in its collinear form a domain wall has a relative energy minimum and remains stable up to a second critical value $\lambda=\lambda_{\mathrm{c}}=3 / 2$. As Eq. (3.8) implies, at this point the frequencies $\omega_{ \pm}$have imaginary increments which lead to a modulational instability in the corresponding antisymmetric modes and in the collinear DB structure, itself. Figure $1 \mathrm{~b}$ shows that the deviations in the directions of the magnetic moments in a canted DB are antisymmetric with respect to its center, which is consistent with an increase in precisely the antisymmetric internal mode.

\section{SPECTRUM OF THE EIGENSTATES OF AN AFM CHAIN WITH A CANTED DOMAIN BOUNDARY}

We now examine the internal mode spectrum of domain walls in the canted phase which arise for values of the exchange interaction greater than critical $\left(\lambda>\lambda_{b}\right)$ and are transformed at this point from the corresponding frequency dependences of the internal modes for $\lambda<\lambda_{\mathrm{b}}$. It was not possible to find an exact analytic solution for the distribution of the magnetization in a discrete canted AFM chain, but near the critical value $\lambda=\lambda_{\mathrm{b}}$ this distribution is given approximately by Eq. (2.8).

For a noncollinear structure Eq. (1.3) is conveniently rewritten in terms of the components $S_{n}^{i}(i=x, y)$ as

$$
\frac{d S_{n}^{x}}{d t}-\lambda\left(S_{n}^{y} m_{n+1}+S_{n}^{y} m_{n-1}-S_{n+1}^{y} m_{n}-S_{n-1}^{y} m_{n}\right)+S_{n}^{y} m_{n}=0,
$$

$$
-\frac{d S_{n}^{y}}{d t}-\lambda\left(S_{n}^{x} m_{n+1}+S_{n}^{x} m_{n-1}-S_{n+1}^{x} m_{n}-S_{n-1}^{x} m_{n}\right)+S_{n}^{x} m_{n}=0
$$

where $m_{n}=\sqrt{1-\left(S_{n}^{x}\right)^{2}-\left(S_{n}^{y}\right)^{2}}$. For concreteness, we assume that magnetization reversal in the static DB takes place in the $X Z$ plane. Then its configuration is determined by $S_{n}^{x(0)}$ $=-S_{-n}^{x(0)}=(-1)^{n} \sqrt{2 \varepsilon} 3^{1-n}$ and $S_{n}^{y(0)}=0$. Recall that the amplitude of this solution was found in the second approximation of perturbation theory, but the way the deviations in the magnetization fall off was determined through the first (principal) approximation. Since these deviations fall off exponentially with the number $n$, in order to solve the dynamic problem to the same approximation and with the same degree of accuracy, we retain the nonlinear terms $\left(S_{ \pm 1}^{x(0)}=\mp \sqrt{2 \varepsilon}\right)$ in the equations (4.1) and (4.2) for the dynamics only with numbers $n=-1,1$, linearizing them afterward for all the remaining moments with respect to small increments in the static solution (2.8):

$$
S_{n}^{x}=S_{n}^{x(0)}+W_{n}(t), \quad S_{n}^{y}=V_{n}(t), \quad|n| \geqslant 2,
$$

where $W_{n}(t), V_{n}(t) \ll S_{n}^{x(0)}$. As opposed to a collinear domain wall, where the magnetic moments rotate along a cone, in a canted boundary the amplitudes of the oscillations in the moments in different directions are different, and the solution has the form

$$
V_{n}=A_{n} \sin (\omega t), \quad W_{n}=B_{n} \cos (\omega t) .
$$

Since the rotation amplitudes of the moments with different parities are different, we write the spatially decreasing solution of the system of Eqs. (4.1) and (4.2) on the $n>0$ semiaxis in the form

$$
\begin{aligned}
& A_{2 m}=c \exp (-2 m \xi), \quad A_{2 m+1}=d \exp (-(2 m+1) \xi), \\
& B_{2 m}=a \exp (-2 m \xi), \quad B_{2 m+1}=b \exp (-(2 m+1) \xi),
\end{aligned}
$$

where the localization parameter $\xi$ is determined by Eq. (3.6), i.e.,

$$
\exp (-\xi)=\left(\sqrt{(1+2 \lambda)^{2}-\omega^{2}}-\sqrt{(1+4 \lambda)-\omega^{2}}\right) / 2 \lambda
$$

Substituting Eqs. (4.3)-(4.5) in Eqs. (4.1) and (4.2) yields the final system of algebraic equations. (Recall that we are including the static cants of the magnetization vectors only for spins with numbers $n= \pm 1$ owing to the rapid decrease in the corresponding amplitudes. As for the dynamic deviations in the local modes, as an example we note that in modes with frequencies adjacent to the continuum spectrum, the amplitudes of the spins with $n= \pm 2$ are significantly greater than the amplitudes with $n= \pm 1$, as shown below.) The equations for $n=1,2$, which play the role of a "boundary condition," reduce to

$$
\begin{aligned}
& \omega a+[(1+2 \lambda)-\lambda \varepsilon] c+2 \lambda \cosh \xi d=0, \\
& \omega c+[(1+2 \lambda)-\lambda \varepsilon] a+2 \lambda \cosh \xi b=0, \\
& \omega b-[(1 \pm \lambda)-(1-\lambda \pm \lambda) \varepsilon] d-\lambda(1-\varepsilon) \exp (-\xi) c=0,
\end{aligned}
$$




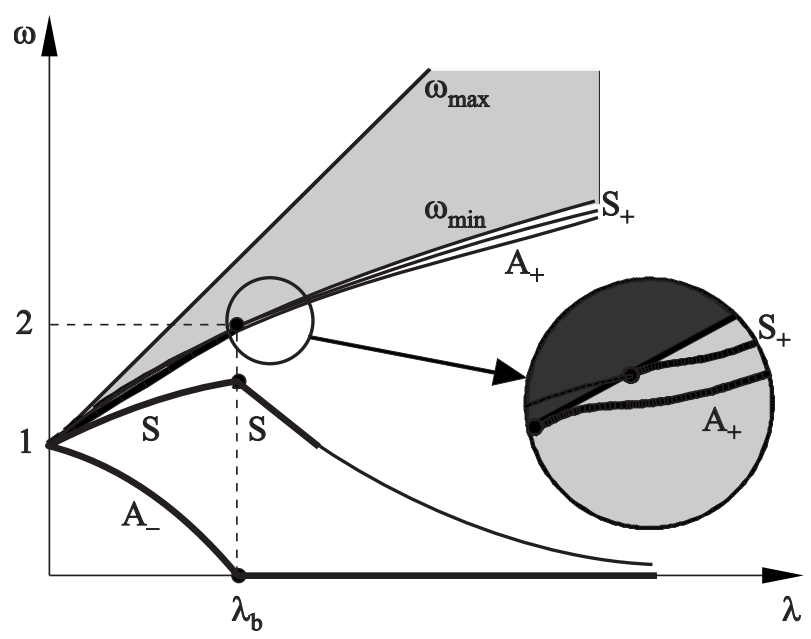

FIG. 4. Frequency dependences on the discreteness parameter for domain wall internal modes in the stability regions for different DB configurations.

$\omega d-[(1 \pm \lambda)-3(1-\lambda \pm \lambda) \varepsilon] b-\lambda(1-\varepsilon) \exp (-\xi) a=0$,

where the \pm signs refer, respectively, to the symmetric and antisymmetric modes. Here the equation for the spectrum of the local modes takes the form

$$
\begin{aligned}
{[1} & \pm \lambda-\omega-2 \varepsilon(1-\lambda \pm \lambda)](1+2 \lambda+\omega-\lambda \varepsilon) \\
& =\lambda^{2}(1-\varepsilon)[1+\exp (-2 \xi)] .
\end{aligned}
$$

It is easy to show that in a symmetric local mode with frequencies detached at the bifurcation point from a frequency $\omega=\sqrt{5 / 2}$, the amplitudes of the oscillations fall off monotonically with the number $\mathrm{n}(\mathrm{a} \approx / 2)$ and the asymptote of the analytic frequency dependence for $\varepsilon \ll 1$ is given by

$$
\omega \approx \sqrt{5 / 2}-(13-\sqrt{10})\left(\lambda-\lambda_{b}\right) / 8 \approx \sqrt{5 / 2}-1.23\left(\lambda-\lambda_{b}\right) .
$$

The frequency of the antisymmetric mode $A_{-}$at the bifurcation point goes to zero and for $\lambda>\lambda_{b}$ turns into a specific shear mode with identically zero frequency. This is related to the fact that in the easy-axis antiferromagnet being examined here, the choice of the plane of magnetization reversal in the static configuration of the canted DB is arbitrary.

Finally, the antisymmetric mode $A_{+}$detaches weakly from the lower boundary of the continuous spectrum at the point $(\omega=2, \lambda=3 / 4)$ :

$$
\omega \approx 2+\varepsilon-\varepsilon^{2}\left[(13 / 12)^{2}+1\right] / 4 \approx 2+\varepsilon-\varepsilon^{2} / 2,
$$

i.e., it detaches from the boundary of the spectrum as $\omega_{\text {min }}(\lambda)-\omega_{-}(\lambda) \approx \varepsilon^{2} / 4$. (Note that, although this effect is $\sim \varepsilon^{2}$, in this case it corresponds to the approximation in which terms $\sim \varepsilon$ were used.)

All of the analytic dependences obtained here are shown in Fig. 4 as thick curves. A comparison of the spectrum of the internal modes in ferro- and antiferromagnets ${ }^{17}$ shows that the internal dynamics of the domain walls is quite different in the two: the number of local modes changes, along with their dependence on the discreteness parameter. In particular, for some modes the frequency does not go to zero at the critical point, but undergoes a sharp bend. However, they have in common that in both cases there is a sharp change in the frequency dependences in the region of $J \sim \beta$, where the frequencies of several local modes descend deeply into the gap of the magnon spectrum and may be experimentally detectable.

The frequency dependences of the internal modes can also be studied in the long wave limit $(\lambda \gg 1)$. The symmetric mode $S$ transforms into low amplitude oscillations of the center of an AFM domain wall in the Peierls potential. This potential is easily found using Indenbom's approach. ${ }^{33} \mathrm{Al}-$ though it only provides a qualitative estimate of the DB energy, the relief, itself, is determined with high accuracy. ${ }^{34}$ It is easy to show that the Peierls DB energy is given by $U$ $=k X_{0}^{2} / 2$, where $X_{0}$ is the position of the center of the domain wall and $k \sim J \exp \left(-\pi^{2} \sqrt{\lambda}\right)$. On the other hand, the effective mass of an AFM DB obeys the proportion $M \sim 1 / J \sqrt{\lambda} .{ }^{30}$ Thus, the frequency of the symmetric internal mode in the long wave limit tends to zero, as $\omega \sim J \sqrt[4]{\lambda} \exp \left(-\pi^{2} \sqrt{\lambda} / 2\right)$ (See Fig. 4.)

The behavior of the antisymmetric mode $A_{+}$in the long wave limit is also easily evaluated. This mode describes an oscillation of the width of the domain wall and, as shown in Refs. 25-28, the splitting off of the antisymmetric internal mode owing to the discreteness of the system depends on the discreteness parameter in the following way: $\omega_{\min }-\omega$ $\sim 1 / \lambda^{2}$ (See Fig. 4.)

In the intermediate range of the discreteness parameter, we have studied the frequency dependences numerically. The results shown in Fig. 4 match well with the analytic results near the bifurcation point $\lambda=3 / 4$ and with the asymptotes of the long wave limit. One interesting feature of the dynamics of AFM domain walls is the appearance of an additional symmetric internal mode, $S_{+}$, which is weakly detached from the continuous magnon spectrum. The frequency of this mode lies above that of the antisymmetric $A_{+}$mode and its frequency dependence "enters" the continuous spectrum when the discreteness parameter $\lambda_{*} \approx 0.84>\lambda_{\mathrm{b}}$. (Similar behavior is observed for the antisymmetric SGE mode.)

\section{CONCLUSION}

All the possible internal modes of essentially discrete domain walls in quasi-two dimensional antiferromagnets have been studied. The existence of a whole spectrum of localized states (symmetric and antisymmetric) has been pointed out and their frequency dependence on the discreteness parameter have been found. It has been shown that in the region where this parameter is on the order of unity, the frequencies of some of the modes split off quite strongly from the linear magnon spectrum and may be observable experimentally. The theoretical results obtained here may be used for explaining experimental data on some new lowdimension magnets.

We thank the editor of the special issue of Low Temperature Physics devoted to the birthday of Academician V. G. Bar'yakhtar for proposing the submission of this material for publication. We would like to point out that V. G. Bar'yakhtar was one of the first to study theoretically the dynamics of quasi-two dimensional antiferromagnets. ${ }^{35}$ 
${ }^{a)}$ Email: kovalev@ilt.kharkov,ua

${ }^{1}$ L. J. De Jongh, W. D. Van Amstel, and A. R. Miedema, Physica (Amsterdam) 58, 277 (1972).

${ }^{2}$ L. I. De Jongh and F. R. Miedema, Adv. Phys. 23, 1 (1974).

${ }^{3}$ L. I. De Jongh, F. C. Batterman, F. R. De Boer, and A. R. Miedema, J. Appl. Phys. 40, 1363 (1969).

${ }^{4}$ H. Arend, K. Tichy, K. Babershke, and F. Rys, Solid State Commun. 18, 999 (1971).

${ }^{5}$ H. Arend, R. Hofmann, and F. Waldner, Solid State Commun. 13, 1629 (1973).

${ }^{6}$ S. Flach and A. V. Gorbach, Phys. Rep. 467, 1 (2008).

${ }^{7}$ K. G. Dergachev, M. I. Kobets, and E. N. Khats'ko, Fiz. Nizk. Temp. 31, 530 (2005) [Low Temp. Phys. 31, 402 (2005)].

${ }^{8}$ M. I. Kobets, K. G. Dergachev, S. L. Gnatchenko, E. N. Khats'ko, Yu. M. Vysochanskii, and M. I. Gurzan, Fiz. Nizk. Temp. 35, 1197 (2009) [Low Temp. Phys. 35, 930 (2009)].

${ }^{9}$ A. A. Stepanov, A. I. Zvyagin, S. V. Volotskii, M. I. Kobets, and V. A. Pashchenko, Fiz. Nizk. Temp. 15, 100 (1989) [Sov. J. Low Temp. Phys. 15, 57 (1989)].

${ }^{10}$ F. Waldner, J. Magn. Magn. Mater. 31-34, 1203 (1983).

${ }^{11}$ F. Waldner, J. Magn. Magn. Mater. 54-77, 873 (1986).

${ }^{12}$ U. T. Schwarz, L. Q. English, and A. J. Sievers, Phys. Rev. Lett. 83, 223 (1999).

${ }^{13}$ A. A. Stepanov, M. I. Kobets, and A. I. Zvyagin, Fiz. Nizk. Temp. 9, 764 (1983) [Sov. J. Low Temp. Phys. 9, 391 (1983)].

${ }^{14}$ A. A. Stepanov, V. A. Pashchenko, and M. I. Kobets, Fiz. Nizk. Temp. 14, 550 (1988) [Sov. J. Low Temp. Phys. 14, 304 (1988)].

${ }^{15}$ A. A. Stepanov, V. A. Pashchenko, and M. I. Kobets, Fiz. Nizk. Temp. 14, 1212 (1988) [Sov. J. Low Temp. Phys. 14, 669 (1988)].

${ }^{16}$ A. A. Stepanov and D. A. Yablonskii, Fiz. Nizk. Temp. 15, 215 (1989) [Sov. J. Low Temp. Phys. 15, 122 (1989)].

${ }^{17}$ A. N. Goncharuk, A. A. Stepanov, and D. A. Yablonskii, Fiz. Tverd. Tela (Leningrad) 31, 132 (1989).

${ }^{18}$ M. V. Gvozdikova, A. S. Kovalev, and Yu. S. Kovshar', Fiz. Nizk. Temp. 24, 635 (1998) [Low Temp. Phys. 24, 479 (1998)].
${ }^{19}$ M. V. Gvozdikova and A. S. Kovalev, Fiz. Nizk. Temp. 25, 1295 (1999) [Low Temp. Phys. 25, 972 (1999)].

${ }^{20}$ M. V. Gvozdikova and A. S. Kovalev, Fiz. Nizk. Temp. 24, 1077 (1998) [Low Temp. Phys. 24, 808 (1998)].

${ }^{21}$ V. G. Bar'yakhtar, B. A. Ivanov, and M. V. Chetkin, Usp. Fiz. Nauk 146, 418 (1985).

${ }^{22}$ V. G. Bar'yakhtar, M. V. Chetkin, B. A. Ivanov, and S. N. Gadetskii, Dynamics of Topological Magnetic Solitons. Experiment and Theory. Tracts in Modern Physics, Springer-Verlag (1994), v. 129.

${ }^{23}$ V. G. Bar'yakhtar and B. A. Ivanov, in: Soliton Thermodynamics of LowDimensional Magnets, Sov. Sci. Rev., I. M. Khalatynikov (ed.), 192 pp., Gordon \& Breach Publishing (1992), v. 16, No. 3.

${ }^{24}$ O. V. Charkina and M. M. Bogdan, in: Symmetry, Integrability and Geometry: Methods and Applications (2006), v. 2.

${ }^{25}$ Yu. S. Kivshar, D. E. Pelinovsky, T. Cretegny, and M. Peyrard, Phys. Rev. Lett. 80, 5032 (1998).

${ }^{26}$ P. G. Kevrekidis and C. K. R. T. Jones, Phys. Rev. E 61, 3114 (2000).

${ }^{27}$ O. M. Braun, Yu. S. Kivshar, and M. Peyrard, Photonics Spectra 56, 6050 (1997).

${ }^{28}$ J. E. Prilepsky and A. S. Kovalev, Phys. Rev. E 71, 046601 (2005).

${ }^{29}$ M. M. Bogdan, A. M. Kosevich, and V. P. Voronov, in: Solitons and Applications, Singapore, World Scientific (1990), p. 231.

${ }^{30}$ A. M. Kosevich, B. A. Ivanov, and A. S. Kovalev, Nonlinear Magnetization Waves. Dynamic and Topological Solitons [in Russian], Naukova Dumka, Kiev (1988); A. M. Kosevich, B. A. Ivanov, and A. S. Kovalev, Phys. Rep. 194, 117 (1990).

${ }^{31}$ J. E. Prilepsky, A. S. Kovalev, M. Johansson, and Yu. S. Kivshar, Phys. Rev. B74, 132404 (2006).

${ }^{32}$ A. M. Kosevich and A. S. Kovalev, Introduction to Nonlinear Physical Mechanics [in Russian], Naukova Dumka, Kiev (1989).

${ }^{33}$ V. L. Indenbom, Kristallografiya 3, 195 (1958).

${ }^{34}$ O. V. Usatenko, A. V. Gorbach, and A. S. Kovalev, Fiz. Tverd. Tela (Leningrad) 43, 1202 (2001).

${ }^{35}$ V. G. Bar'yakhtar, A. I. Zvyagin, M. I. Kobets, V. N. Krivoruchko, A. A. Stepanov, and D. A. Yablonskii, Fiz. Nizk. Temp. 11, 1113 (1985) [Sov. J. Low Temp. Phys. 11, 615 (1985)].

Translated by D. H. McNeill 